\title{
Analytical Relation between Quark Confinement and Chiral Symmetry Breaking in QCD
}

\author{
Hideo Suganuma*, Takahiro M. Doi \\ Department of Physics \& Division of Physics and Astronomy, Graduate School of Science, \\ Kyoto University, Kitashirakawaoiwake, Sakyo, Kyoto 606-8502, Japan \\ E-mail: suganuma@scphys.kyoto-u.ac.jp
}

\section{Takumi Iritani}

High Energy Accelerator Research Organization (KEK), Tsukuba, Ibaraki 305-0801, Japan

\begin{abstract}
We study the relation between quark confinement and spontaneous chiral-symmetry breaking directly in QCD. In lattice QCD formalism, we derive an analytical gauge-invariant relation between the Polyakov loop $\left\langle L_{P}\right\rangle$ and the Dirac eigenvalues $\lambda_{n}$, i.e., $\left\langle L_{P}\right\rangle \propto \sum_{n} \lambda_{n}^{N_{t}-1}\left\langle n\left|\hat{U}_{4}\right| n\right\rangle$, on a temporally odd-number lattice, where the temporal lattice size $N_{t}$ is odd. Here, $|n\rangle$ denotes the Dirac eigenmode, i.e., $\not D|n\rangle=i \lambda_{n}|n\rangle$, and $\hat{U}_{4}$ the temporal link-variable operator. We here use an ordinary square lattice with the normal periodic boundary condition for link-variables $U_{\mu}(s)$ in the temporal direction. Because of the factor $\lambda_{n}^{N_{t}-1}$ in the analytical relation, the contribution of low-lying Dirac modes to the Polyakov loop is negligibly small in both confined and deconfined phases, while the low-lying Dirac modes are essential for chiral symmetry breaking. Also, in lattice QCD simulations, we numerically confirm the analytical relation, non-zero finiteness of $\left\langle n\left|\hat{U}_{4}\right| n\right\rangle$ for each Dirac mode, and negligibly small contribution of low-lying Dirac modes to the Polyakov loop. Thus, we conclude that low-lying Dirac modes are not essential for confinement, which indicates no direct one-to-one correspondence between confinement and chiral symmetry breaking in QCD.
\end{abstract}

XV International Conference on Hadron Spectroscopy

4-8/11/2013

Nara, Japan

${ }^{*}$ Speaker. 


\section{Introduction: Are color confinement and CSB one-to-one in QCD?}

QCD has two outstanding nonperturbative phenomena of color confinement and spontaneous chiral-symmetry breaking (CSB) [1]. However, their relation is not yet known directly from QCD, and to clarify their precise relation is one of the important problems in theoretical physics [2, 3, 4].

For quark confinement, the Polyakov loop $\left\langle L_{P}\right\rangle$ is one of the typical order parameters, and it relates to the single-quark free energy $E_{q}$ as $\left\langle L_{P}\right\rangle \propto e^{-E_{q} / T}$ at temperature $T$. Also, its fluctuation is recently found to be important in the QCD phase transition [5]. For CSB, the order parameter is the chiral condensate $\langle\bar{q} q\rangle$, and low-lying Dirac modes play the essential role [6].

A strong correlation between confinement and CSB has been suggested by almost coincidence between deconfinement and chiral-restoration temperatures [7], although slight difference of about $25 \mathrm{MeV}$ between them is pointed out in recent lattice QCD studies [8]. Their correlation has been also suggested in terms of QCD-monopoles [2, 3], which topologically appear in QCD in the maximally Abelian gauge. By removing the monopoles from the QCD vacuum, confinement and CSB are simultaneously lost in lattice QCD [2] 3]. (See Fig.1.) This indicates an important role of QCD-monopoles to both confinement and CSB, and thus these two phenomena seem to be related via the monopole. However, the direct relation of confinement and CSB is still unclear.

Then, we have a question. if only the relevant ingredient of CSB is carefully removed from the QCD vacuum, how will be quark confinement? In this study, we derive an analytical relation between the Polyakov loop and the Dirac modes in temporally odd-number lattice QCD, where the temporal lattice size is odd, and discuss the relation between confinement and CSB [9 10].
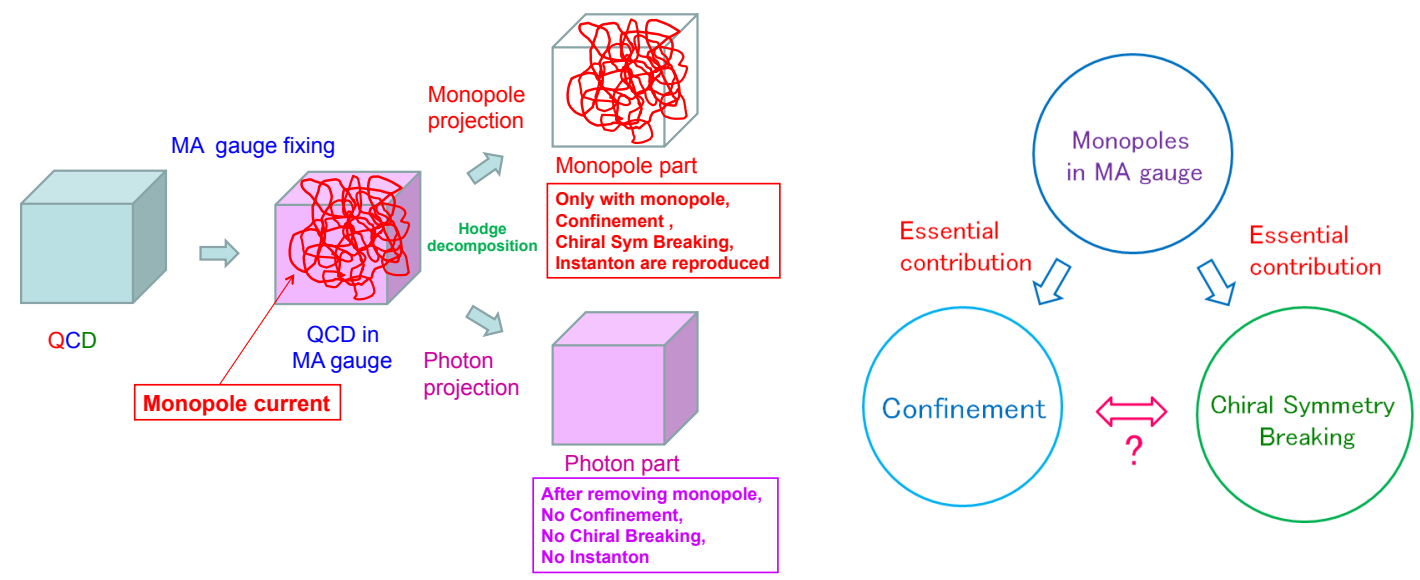

Figure 1: In the MA gauge, QCD-monopoles topologically appear. By removing the monopole from the QCD vacuum, confinement and CSB are simultaneously lost [2] 3]. This means essential role of monopoles to both confinement and CSB. However, the direct relation between confinement and CSB is unclear.

\section{Lattice QCD formalism for Dirac operator, Dirac eigenvalues and Dirac modes}

Note that, in our studies 910 , we just consider the mathematical expansion by eigenmodes $|n\rangle$ of the Dirac operator $\not D=\gamma_{\mu} D_{\mu}$, using the completeness of $\sum_{n}|n\rangle\langle n|=1$. In general, instead of $\not D$, one can consider any (anti)hermitian operator, e.g., $D^{2}=D_{\mu} D_{\mu}$, and the expansion in terms of its eigenmodes. In this paper, to consider CSB, we adopt $\not D$ and the expansion by its eigenmodes. 
We use an ordinary square lattice with spacing $a$ and size $V \equiv N_{s}^{3} \times N_{t}$, and impose the normal periodic boundary condition for the link-variable $U_{\mu}(s)=\mathrm{e}^{i a g A_{\mu}(s)}\left(A_{\mu}\right.$ : gluon fields) in the temporal direction. In lattice QCD, the Dirac operator $\not D=\gamma_{\mu} D_{\mu}$ is expressed with $U_{\mu}(s)$ as

$$
\not D_{s, s^{\prime}} \equiv \frac{1}{2 a} \sum_{\mu=1}^{4} \gamma_{\mu}\left[U_{\mu}(s) \delta_{s+\hat{\mu}, s^{\prime}}-U_{-\mu}(s) \delta_{s-\hat{\mu}, s^{\prime}}\right]
$$

where $\hat{\mu}$ is the unit vector in $\mu$-direction in the lattice unit, and $U_{-\mu}(s) \equiv U_{\mu}^{\dagger}(s-\hat{\mu})$. Adopting hermitian $\gamma$-matrices as $\gamma_{\mu}^{\dagger}=\gamma_{\mu}$, the Dirac operator $\not D$ is anti-hermitian and satisfies $\not \not_{s^{\prime}, s}^{\dagger}=-\not D_{s, s^{\prime}}$. We introduce the normalized Dirac eigen-state $|n\rangle$ and the Dirac eigenvalue $i \lambda_{n}\left(\lambda_{n} \in \mathbf{R}\right)$,

$$
\not D|n\rangle=i \lambda_{n}|n\rangle, \quad\langle m \mid n\rangle=\delta_{m n}, \quad \sum_{n}|n\rangle\langle n|=1 .
$$

The Dirac eigenfunction $\psi_{n}(s) \equiv\langle s \mid n\rangle$ satisfies $\sum_{s^{\prime}} \not D_{s, s^{\prime}} \psi_{n}\left(s^{\prime}\right)=i \lambda_{n} \psi_{n}(s)$ and gauge-transforms as $\psi_{n}(s) \rightarrow V(s) \psi_{n}(s)$, which is the same as the quark field, apart from an irrelevant global phase [4].

Now, we define the link-variable operator $\hat{U}_{ \pm \mu}$ [4] by the matrix element of

$$
\left\langle s\left|\hat{U}_{ \pm \mu}\right| s^{\prime}\right\rangle=U_{ \pm \mu}(s) \delta_{s \pm \hat{\mu}, s^{\prime}} .
$$

With the link-variable operator, the Dirac operator and covariant derivative are simply expressed,

$$
\hat{\not D}=\frac{1}{2 a} \sum_{\mu=1}^{4} \gamma_{\mu}\left(\hat{U}_{\mu}-\hat{U}_{-\mu}\right), \quad \hat{D}_{\mu}=\frac{1}{2 a}\left(\hat{U}_{\mu}-\hat{U}_{-\mu}\right)
$$

The Polyakov loop is also simply written as the functional trace of $\hat{U}_{4}^{N_{t}}$, i.e., $\left\langle L_{P}\right\rangle=\frac{1}{N_{c} V}\left\langle\operatorname{Tr}_{c}\left\{\hat{U}_{4}^{N_{t}}\right\}\right\rangle$, where, " $\operatorname{Tr}_{c}$ " denotes the functional trace of $\operatorname{Tr}_{c} \equiv \sum_{s} \operatorname{tr}_{c}$ with the trace $\operatorname{tr}_{c}$ over color index.

\section{Direct relation between Polyakov loop and Dirac modes on odd-number lattice}

Now, we consider a temporally odd-number lattice [9 10, where the temporal lattice size $N_{t}$ is odd. The spatial lattice size $N_{s}$ is taken to be larger than $N_{t}$, i.e., $N_{s}>N_{t}$. Note that, in the continuum limit of $a \rightarrow 0$ and $N_{t} \rightarrow \infty$, any number of large $N_{t}$ gives the same physical result. Then, it is no problem to use the odd-number lattice.

In general, only gauge-invariant quantities such as closed loops and the Polyakov loop survive in QCD, according to the Elitzur theorem [7]. All the non-closed lines are gauge-variant and their expectation values are zero. Note here that any closed loop needs even-number link-variables on the square lattice, except for the Polyakov loop. (See Fig.2.) On the temporally odd-number lattice, we consider the following functional trace and its expectation value [101:

$$
I \equiv \operatorname{Tr}_{c, \gamma}\left(\hat{U}_{4} \hat{\not}^{N_{t}-1}\right), \quad\langle I\rangle=\left\langle\operatorname{Tr}_{c, \gamma}\left(\hat{U}_{4} \hat{\not}^{N_{t}-1}\right)\right\rangle .
$$

Here, $\operatorname{Tr}_{c, \gamma} \equiv \sum_{s} \operatorname{tr}_{c} \operatorname{tr}_{\gamma}$ includes $\operatorname{tr}_{c}$ and the trace $\operatorname{tr}_{\gamma}$ over spinor index. The expectation value $\langle I\rangle$ is obtained as the gauge-configuration average in lattice QCD. In the case of enough large volume $V$, one can expect $\langle O\rangle \simeq \operatorname{Tr} O / \operatorname{Tr} 1$ for any operator $O$ at each gauge configuration.

From Eq.2.4,$\hat{U}_{4} \hat{D}^{N_{t}-1}$ is expressed as a sum of products of $N_{t}$ link-variable operators, since the Dirac operator $\hat{\not}$ includes one link-variable operator in each direction of $\pm \mu$. Then, $\hat{U}_{4} \hat{D}^{N_{t}-1}$ 


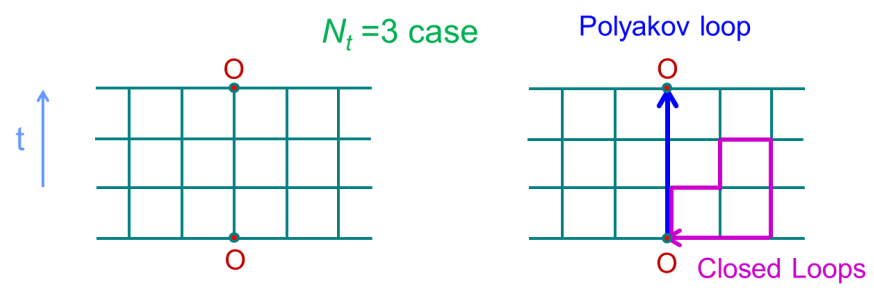

Figure 2: An example of the temporally odd-number lattice. Only gauge-invariant quantities such as closed loops and the Polyakov loop survive in QCD. Closed loops have even-number links on the square lattice.
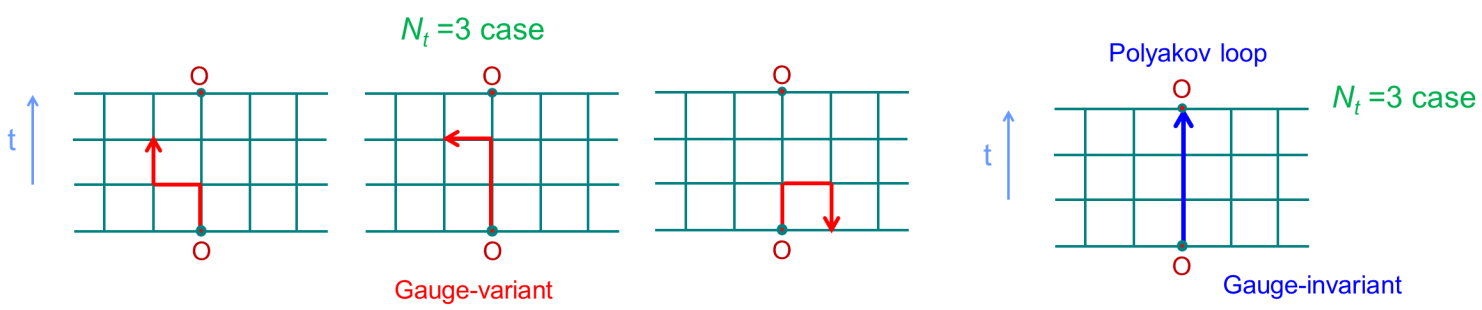

Figure 3: Examples of the trajectories stemming from $\langle I\rangle=\left\langle\operatorname{Tr}_{c, \gamma}\left(\hat{U}_{4} \hat{D}^{N_{t}-1}\right)\right\rangle$. For each trajectory, the total length is $N_{t}$, and the "first step" is positive temporal direction corresponding to $\hat{U}_{4}$. All the trajectories with the odd-number length $N_{t}$ cannot form a closed loop on the square lattice, so that they are gauge-variant and give no contribution, except for the Polyakov loop. Thus, only the Polyakov-loop ingredient survives in $\langle I\rangle$.

includes many trajectories with the total length $N_{t}$ (in the lattice unit) on the square lattice, as shown in Fig.3. Note that all the trajectories with the odd-number length $N_{t}$ cannot form a closed loop on the square lattice, and thus give gauge-variant contribution, except for the Polyakov loop.

Hence, among the trajectories stemming from $\langle I\rangle=\left\langle\operatorname{Tr}_{c, \gamma}\left(\hat{U}_{4} \hat{D}^{N_{t}-1}\right)\right\rangle$, all the non-loop trajectories are gauge-variant and give no contribution, according to the Elitzur theorem [7]. Only the exception is the Polyakov loop. (See Fig.3.) Thus, in $\langle I\rangle$, only the Polyakov-loop ingredient can survive as the gauge-invariant quantity, and $\langle I\rangle$ is proportional to the Polyakov loop $\left\langle L_{P}\right\rangle$.

Actually, we can mathematically derive the following relation [9]:

$$
\begin{aligned}
\langle I\rangle & =\left\langle\operatorname{Tr}_{c, \gamma}\left(\hat{U}_{4} \hat{D}^{N_{t}-1}\right)\right\rangle=\left\langle\operatorname{Tr}_{c, \gamma}\left\{\hat{U}_{4}\left(\gamma_{4} \hat{D}_{4}\right)^{N_{t}-1}\right\}\right\rangle & & (\because \text { only gauge-invariant terms survive }) \\
& =4\left\langle\operatorname{Tr}_{c}\left(\hat{U}_{4} \hat{D}_{4}^{N_{t}-1}\right)\right\rangle & & \left(\because \gamma_{4}^{N_{t}-1}=1, \operatorname{tr}_{\gamma} 1=4\right) \\
& =\frac{4}{(2 a)^{N_{t}-1}}\left\langle\operatorname{Tr}_{c}\left\{\hat{U}_{4}\left(\hat{U}_{4}-\hat{U}_{-4}\right)^{N_{t}-1}\right\}\right\rangle & & \left(\because \hat{D}_{4}=\frac{1}{2 a}\left(\hat{U}_{4}-\hat{U}_{-4}\right)\right) \\
& =\frac{4}{(2 a)^{N_{t}-1}}\left\langle\operatorname{Tr}_{c}\left\{\hat{U}_{4}^{N_{t}}\right\}\right\rangle=\frac{12 V}{(2 a)^{N_{t}-1}}\left\langle L_{P}\right\rangle . & & (\because \text { only gauge-invariant terms survive })(3.2
\end{aligned}
$$

On the other hand, we calculate the functional trace in Eq. 3.1) using the complete set of the Dirac-mode basis $|n\rangle$ satisfying $\sum_{n}|n\rangle\langle n|=1$, and find the Dirac-mode representation of

$$
\langle I\rangle=\sum_{n}\left\langle n\left|\hat{U}_{4} \hat{D}^{N_{t}-1}\right| n\right\rangle=i^{N_{t}-1} \sum_{n} \lambda_{n}^{N_{t}-1}\left\langle n\left|\hat{U}_{4}\right| n\right\rangle .
$$

Combing Eqs. (3.2) and (3.3), we obtain the analytical relation between $\left\langle L_{P}\right\rangle$ and $\lambda_{n}$ in QCD [9, 10]:

$$
\left\langle L_{P}\right\rangle=\frac{(2 a i)^{N_{t}-1}}{12 V} \sum_{n} \lambda_{n}^{N_{t}-1}\left\langle n\left|\hat{U}_{4}\right| n\right\rangle .
$$


This is a direct relation between the Polyakov loop $\left\langle L_{P}\right\rangle$ and the Dirac modes in QCD, and is mathematically valid on the temporally odd-number lattice in both confined and deconfined phases. From Eq. 3.47, we can investigate each Dirac-mode contribution to the Polyakov loop individually.

Remarkably, due to the factor $\lambda_{n}^{N_{t}-1}$, low-lying Dirac-mode contribution is negligibly small in RHS of Eq. (3.4), compared to the other Dirac-mode contribution. In fact, the low-lying Dirac modes give little contribution to the Polyakov loop, regardless of confined or deconfined phase [9].

Here, we give several comments on the relation (3.4) in order.

1. Eq. (3.4) is manifestly gauge invariant, because $\left\langle n\left|\hat{U}_{4}\right| n\right\rangle=\sum_{s}\langle n \mid s\rangle\left\langle s\left|\hat{U}_{4}\right| s+\hat{t}\right\rangle\langle s+\hat{t} \mid n\rangle=$ $\sum_{s} \psi_{n}^{\dagger}(s) U_{4}(s) \psi_{n}(s+\hat{t})$ is gauge invariant under the gauge transformation, $\psi_{n}(s) \rightarrow V(s) \psi_{n}(s)$.

2. In RHS of Eq. 3.4, there is no cancellation between chiral-pair Dirac eigen-states, $|n\rangle$ and $\gamma_{5}|n\rangle$, because $\left(N_{t}-1\right)$ is even, i.e., $\left(-\lambda_{n}\right)^{N_{t}-1}=\lambda_{n}^{N_{t}-1}$, and $\left\langle n\left|\gamma_{5} \hat{U}_{4} \gamma_{5}\right| n\right\rangle=\left\langle n\left|\hat{U}_{4}\right| n\right\rangle$.

3. The relation (3.4) is correct regardless of presence or absence of dynamical quarks, although dynamical quark effects appear in $\left\langle L_{P}\right\rangle$, the Dirac eigenvalue distribution $\rho(\lambda)$ and $\left\langle n\left|\hat{U}_{4}\right| n\right\rangle$.

4. The relation (3.4) is correct also at finite density and temperature for any color number $N_{c}$.

In lattice QCD simulations, we also numerically confirm Eq. (3.4) and quite small contribution of low-lying Dirac modes to the Polyakov loop in both confined and deconfined phases [9 10].

From the analytical relation (3.4) and the numerical confirmation, we conclude that low-lying Dirac-modes give negligibly small contribution to the Polyakov loop, and are not essential for confinement, while these modes are essential for chiral symmetry breaking. This conclusion indicates no direct one-to-one correspondence between confinement and chiral symmetry breaking in QCD.

\section{Acknowledgements}

H.S. and T.I. are supported in part by the Grant for Scientific Research [(C) No.23540306, E01:21105006, No.21674002] from the Ministry of Education, Science and Technology of Japan.

\section{References}

[1] Y. Nambu and G. Jona-Lasinio, Phys. Rev. 122 (1961) 345; Phys. Rev. 124 (1961) 246.

[2] H. Suganuma, S. Sasaki and H. Toki, Nucl. Phys. $B 435$ (1995) 207; Prog. Theor. Phys. 94 (1995) 373.

[3] O. Miyamura, Phys. Lett. 3353 (1995) 91; R.M. Woloshyn, Phys. Rev. D51 (1995) 6411.

[4] S. Gongyo, T. Iritani and H. Suganuma, Phys. Rev. D86 (2012) 034510; T. Iritani and H. Suganuma, arXiv:1305.4049[hep-lat]; PoS (Lattice 2012) (2012) 218; PoS (Confinement X) (2013) 053.

[5] P.M.Lo, B.Friman, O.Kaczmarek, K. Redlich, C. Sasaki, Phys. Rev. D88 (2013) 014506; ibid. 074502.

[6] T. Banks and A. Casher, Nucl. Phys. B169 (1980) 103.

[7] H.-J. Rothe, Lattice Gauge Theories, 4th edition, World Scientific, 2012, and its references.

[8] Y. Aoki et al., Phys. Lett. B643 (2006) 46; Nature 443 (2006) 675.

[9] H. Suganuma, T.M. Doi and T. Iritani, PoS (Lattice 2013) (2013) 374; PoS (QCD-TNT-III) (2014) 042; Eur. Phys. J. Web of Conference (ICNFP2013) (2014).

[10] T.M. Doi, H. Suganuma, T. Iritani, PoS (Lattice 2013) (2013) 375; PoS (Hadron 2013) (2013) 122. 